\title{
Ontology for Crop Management-A Core Vocabulary of Agricultural Activity
}

\author{
V. Anandhi*, J. Venkitapirabu, S. K. Natarajan and C. S. Sumathi
}

Tamil Nadu Agricultural University, Coimbatore, India

*Corresponding author

\section{A B S T R A C T}

\section{Keywords}

Ontology, Crop management, Instance, Class, Repository

Article Info

Accepted:

24 August 2020

Available Online:

10 September 2020
Ontology is an explicit specification of a conceptualization". This paper describes the construction of ontology for the agricultural domain, and the crop management in particular. Ontology together with a set of individual instances of classes constitutes a knowledge base. TNAUCrpOnt is the proposed ontology tool used for facilitating indexing and searching processes in a repository environment. This repository contains information in the form of instances. It contains various concepts related to the crop management. A user friendly Ontology is developed for farmers, scientists and students for accessing information with more accuracy and clarity.

\section{Introduction}

Agriculture is the backbone of the economic system in the country. In addition to providing food and raw materials, agriculture provides employment opportunities to about $70 \%$ of the population, either directly or indirectly (1). For most developing countries and in some developed countries, agricultural products constitute the major items for international trade. Thus, agriculture is an important domain for research. Database is a structured collection of interrelated data which provides a way to store and retrieve information conveniently and efficiently (2). The data are maintained as tables. In a database, to relate one table with another, concept of keys is used. Ontology together with a set of individual instances of classes constitutes a knowledge base. In reality, there is a fine line where the ontology ends and the knowledge base begins.

In Ontology, Knowledge collection and representation is in a usable and efficient way is quiet cumbersome, time consuming and a huge process. Sharing of the same knowledge in different applications and its reuse without or little modifications in solving separate problems is a critical factor in knowledge aggregation and dissemination (3). Among knowledge representation techniques, 
ontology formalization can be used to model real world in a consistent, formal, manageable and reusable way (4). Ontologies can be used to facilitate several aspects of knowledge management such as knowledge representation, knowledge sharing and reuse, knowledge evolution, knowledge classification and knowledge search and retrieval (5). Furthermore, ontologies provide improved communication among humans, better documentation, improved maintainability, reliability and interoperability. Ontologies provide a disciplined and structured way to describe knowledge domains and represent their knowledge.

Ontology was originally a term used in philosophy, which is the study of the kind of things that exist (6). Tom Gruber (1993) of Stanford University was the first to formally define Ontology (7). He defined Ontology as "an explicit specification of a conceptualization." It can be used to model a domain formally so that it can be used for knowledge sharing and information integration. As information service has passed the stage of data and information management and entered the era of knowledge management, ontology has gradually become an important tool of knowledge management through providing a shared conceptual model to express knowledge. In comparison with traditional knowledge management methods, Ontology based knowledge management are better suited to meet today's applications requirements.

In the field of agricultural information management, ontology has been proven to have wide applications in the classification of agricultural information, the construction of information and knowledge database, the development and research of intelligent search engine, the realization of cooperative information service etc (8).
There are numerous Agriculture-based ontology projects that have been undertaken and developed with co-operation from various countries. AGROVOC, a Global Agriculture Ontology Applications is a controlled vocabulary created in 1980 , by Food and Agriculture Organization (FAO), UN. FAO wanted to develop a new model for AGROVOC which would be more refined and precise. Agricultural Ontology Service (AOS) was created for utilization of AGROVOC thesaurus at its core. World Agriculture Information Centre (WAICENT) is a multilingual knowledge management system, powered by FAO. FAO knowledge of agriculture is available to users around the world through the WAICENT portal. Keyword search is available in WAICENT (9). Crop Ontology (CO) is the genius of the Generation Challenge Program (GCP). Agricultural crop databases maintained in gene banks of the Consultative Group on International Agricultural Research (CGIAR) are valuable sources of information for researchers, information managers, breeders etc. Ontologies for each crop are constructed and maintained by the GCP $\mathrm{CO}$ web portal (10).Plant Ontology (PO) is a detailed vocabulary and database store that links plant anatomy, morphology, growth and development to plant genomics data (11).

A controlled vocabulary is a collection of terms organised in a hierarchy intended to serve as a standard nomenclature (12). TNAUCrpOnt ontology was the result of this endeavor. It uses concepts to encapsulate meanings associated with terms to define their properties. TNAUCrpOnt contains knowledge concerning cultivation techniques, pest management and crop physiology. Ontology has been extended to include knowledge about propagation, post-harvest physiology, consumption and marketing. Using the proposed ontology, users can extract useful knowledge on crop species cultivated in 
various agro climate and diverse land use management systems. The objective is to describe the specific domain taking into consideration the usage of the ontology and to provide enough information to use ontology in specific to crop management of Tamil Nadu. The capability for knowledge indexing and searching is a crucial factor in applications. The system would return results for the user's request in related concepts. The system helps the users to explore and clarify what they actually need and find useful relevant information.

Fig.1 Angular and Spring Boot Architecture

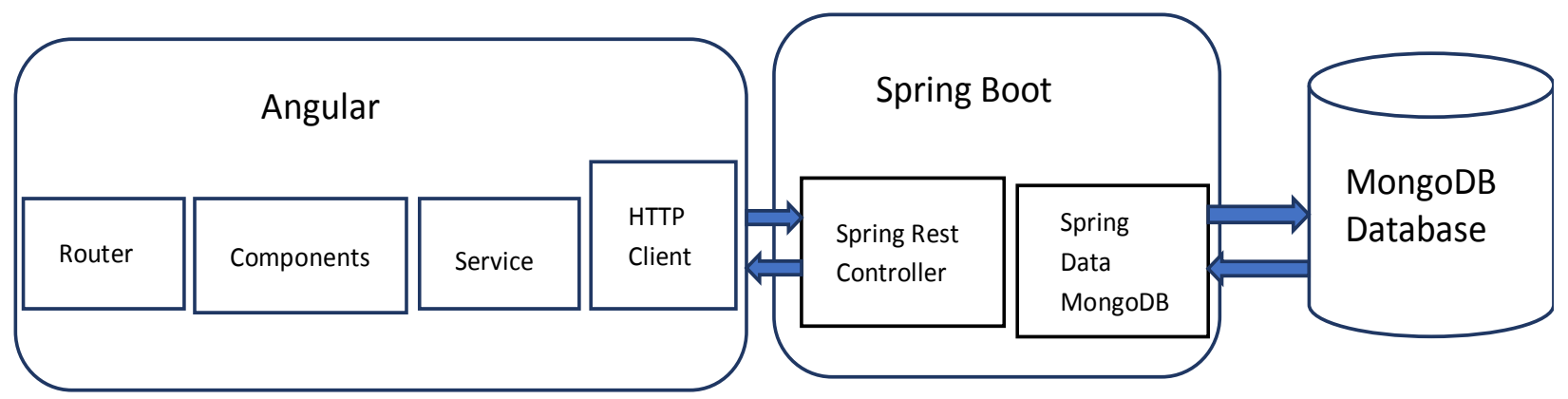

Subsequently the concept is split into more concrete concepts. When farmers plan or do a certain agricultural activity, the first decision is what for they would take the action, i.e., purpose is the first attribute to distinguish agriculture activities. After the purpose is well specified, we use other attributes, i.e., act (type of action), target, place, means, equipment, and season in the order of succession. Thus the management activity of the crop is defined. These eight attributes are used to define the concept and to form the hierarchy of the agricultural activity. Ontology is used to provide the facts and the attributes of the fact-attribute-value structure of the rules. The purpose of ontology determines the relevant knowledge and the knowledge that should be added to ontology and the relations between its components. However, they share a great amount of concepts and usually they follow similar principles in their construction. There are several efforts towards construction and usage of ontologies in several application fields and for different purposes (13). TNAUCrpOnt developed is used for indexing and searching purposes. The ontology describes production of agricultural crops in the area of crop management. The objective of the proposed ontology is the systematic organisation and representation of knowledge and terminology, concerning all stages of production and marketing of these agricultural crops. Ontology development is an evolving process. The translation mechanism should be suitable and flexible enough for this iterative and everchanging process. The technologies used in TNAUCrpOnt for the user interface are Angular, Node JS, Spring boot, Java and MongoDB.

Front-end side is developed with Angular. Spring boot backend server and Spring Data MongoDB for interacting with MongoDB database. Angular is one of the most popular JavaScript frameworks with incredible tooling, speed and performance. Angular Client sends HTTP Requests and retrieve HTTP Responses using axios, shows data on the components. Angular Router is adopted for navigating to pages. Usage of spring boot and angular with routing is used to build the application. Also, we will be integrating Mongo DB database with the backend code. 
Spring Boot interacts with MongoDB Database using Spring Data MongoDB.

Information is stored in the mongo $\mathrm{db}$ as key value pairs. User can access the angular user interface to search key. Spring boot service will act as middleware to communicate the user interface and backend. All these services are contained using docker. While deploying these services we deploy as kubernetes clusters which will take care the spin out and spin in the services of the performance. This is the repository which contains information in the form of instances. It contains various concepts related to the crop management. After identifying the concepts, it is arranged in Taxonomical Hierarchy. The query as input, and processes the query against Expert Knowledge Bank and display the results to the user. The users will be provided with an interface for them to query their search. The Query formulated would be executed by Indexing and Searching. The Users interact with the system. Farmers and Agricultural Information users are the people who finally consume the expert advice or Conceptual information directly from TNAUCropOnt.

In conclusion this paper explains the endeavor taken towards the development of an ontology concerning the agricultural domain and more particularly the crop management. Students can consult the ontology to identify and learn concepts regarding crop management. Growers are able to search for useful knowledge concerning specific cultivation techniques in the field. Moreover, consumers are able to consult the ontology to identify several characteristics of crop management. TNAUCrpOnt can be further extended to cover all the aspects of the related to seed preparation to sowing till post-harvest activities and the Concepts can be represented in different languages as many farmers are familiar only with their regional language.

\section{References}

1. The Importance Of Agriculture Agriculture Goods. Agriculture Goods. N.p., 2017. Web. 26 Apr. 2017.

2. Henry F. Korth and Abraham Silberschatz. Database System Concepts. McGraw-Hill, Inc., New York, NY, USA, 1990.

3. Applying an agricultural ontology to webbased applications Michael T. Maliappis Informatics Laboratory, Agricultural University of Athens, 75 lera Odos street, 11855 Athens, Greece, Int. J. Metadata, Semantics and Ontologies, Vol. 4, Nos. $1 / 2,2009$ page 133

4. Construction of the Ontology-Based Agricultural Knowledge Management System ZHENG Ye-lu, HE Qi-yun, QIAN Ping and LI Ze Institute of Science and Technology Information, Guangdong Academy of Agricultural Sciences, Guangzhou 510640, P.R. China, Journal of Integrative Agriculture 2012, 11(5): 700-709, May 2012.

5. Uschold, M. and Gruninger, M. (2004) 'Ontologies and semantics for seamless connectivity', SIGMOD Record, Vol. 33, No. 3, pp.58-64.

6. B. Chandrasekaran, J. Josephson and V. Benjamins, "What are ontologies, and why do we need them?", IEEE Intelligent Systems, vol. 14, no. 1, pp. 20-26, 1999

7. Gruber, Thomas R. "A Translation Approach to Portable Ontology Specifications". Knowledge Acquisition 5.2 (1993): 199-220. Web.

8. Beck H W, Kim S, Hagan D. 2005. A crop-pest ontology for extension publications. In: Proceedings of 2005 EFITA/ WCCA Joint Congress on IT in Agriculture. Vila Real, EFITA, Portugal. pp. 1169-1176

9. "Waicent - World Agricultural Information Centre Portal", Fao.Org, 2017. (Online). Available: Http://Www.Fao.Org/Waicent/St/Level_1. 
Asp?Main_Id=8. (Accessed: 27- Apr2017).

10. Crop Ontology Curation Tool, Cropontology.org, 2017. (Online). Available: http://www.cropontology.org/. (Accessed: 26- Apr- 2017).

11. O. WG, "Plant Ontology", Obofoundry.org, 2017. Available: http://www.obofoundry.org/ontology/po.ht ml. (Accessed: 26- Apr- 2017).

12. Price, C., O’Neil, M., Bentley, T.E. and
Brown, P.J.B. (1998) 'Exploring the ontology of surgical procedures in the Read Thesaurus', Methods of Information in Medicine, Vol. 37, pp.420-425.

13. Oliver, D.E., Rubin, D.L., Stuart, J.M., Hewett, M., Klein, T.E. and Altman, R.B. (2002) 'Ontology development for a pharmacogenetics knowledge base', Pacific Symposium on Biocomputing, Lihue, Hawaii, Vol. 7, pp.65-76.

\section{How to cite this article:}

Anandhi, V., J. Venkitapirabu, S. K. Natarajan and Sumathi, C. S. 2020. Ontology for Crop Management-A Core Vocabulary of Agricultural Activity. Int.J.Curr.Microbiol.App.Sci. 9(09): 3364-3368. doi: https://doi.org/10.20546/ijcmas.2020.909.418 